\title{
Block Trading Based Volatility Forecasting: An Application of VACD-FIGARCH Model
}

\author{
Teng-Tsai TU*, Chih-Wei LIAO**
}

Received: October 16, 2019 Revised: February 16, 2020 Accepted: March 1, 2020.

\begin{abstract}
The purpose of this study is to construct the ACD model for the block trading volume duration. The ACD model based on the block trading volume duration is referred to as Volume ACD (VACD) in this study. By integrating with GARCH-type models, the VACD based GARCH type models, which include VACD-GARCH, VACD-IGARCH and VACD-FIGARCH models, are set up. This study selects Chunghwa Telecom (CHT) Inc., offering the America Depository Receipt (ADR) in NYSE, to investigate the block trading volume duration in Taiwanese equity market. The empirical results indicate that the long memory in volume duration series increases dependence at level of volatility clustering by VACD (2,1)-FIGARCH $(3, \mathrm{~d}, 1)$ model. Moreover, the VACD $(2,1)$-IGARCH $(1,1)$ exhibits relatively better performance of prediction on capturing block trading volume duration. This volatility model is more appropriate in this study to portray the change of the CHT Inc. prices and provides more information about the volatility process for investment strategy, which can be a reference indicator of financial asset pricing, hedging strategy and risk management.
\end{abstract}

Keywords: Volatility, Volume Duration, ACD, VACD-GARCH, VACD-FIGARCH

JEL Classification Code: C22, C53, F37, G12, G17

\section{Introduction}

In general financial theory, when investors obtain private news and carry on their investment strategy, it would exhibit large amount of transactions. The block trading volume would have a great impact on the change of stock price. In the literature of financial economics and econometrics, most studies focus on daily prices, asset returns and the stochastic process of volatility. Specifically, many studies have adopted the variables of the trading price, trading volume and trading size in empirical analysis. We can consider the trading sizes in modelling the relationships between asset price and volume due to the advantage of

*First Author and Corresponding Author, Graduate Institute of International Business, National Taipei University [Postal Address: 13F., No. 288, Xueqin Rd., Shulin Dist., New Taipei City 238, Taiwan] Email: tttu@gm.ntpu.edu.tw,

**Department of Finance, Ming Chuan University.

Email: g_w_liao@yahoo.com.tw

(c) Copyright: The Author(s)

This is an Open Access article distributed under the terms of the Creative Commons Attribution Non-Commercial License (http://Creativecommons.org/licenses/by-nc/4.0/) which permits unrestricted noncommercial use, distribution, and reproduction in any medium, permits unrestricted noncommercial use,
provided the original work is properly cited. private information captured by investors who would like to trade large quantities. If the market microstructure data were adopted even further in empirical models, the event of block trading volume can be viewed as driving both abnormal returns and volume processes, and hence large trading volume can be viewed as an important explanatory variable in the stochastic process of stock price to conduct empirical analysis.

Previous studies are plausible and even arbitrary to analyze the time series data with econometric techniques of fixed time interval. However, there might be a significant loss of information by adopting this modelling strategy. In reality, the transaction information arriving at irregular times may be informative about the behavior of the traders. Therefore, Engle and Russell (1998) introduce the autoregressive conditional duration (ACD) model to treat the time between events as a stochastic process and to capture more information between the irregular arrival times of trades. The ACD model is a model of transaction volume. The general formulation of GARCH model for irregularly spaced financial data is theoretically more interesting. Thus, Engle (2000) proposes the ACD-GARCH model to capture the volatilities of the asset returns. Based 
on this framework of the ACD-GARCH model, this study tends to find more appropriate time series model to capture the volatility dynamics of financial instrument returns.

Differing from many previous studies, the objective of this study is to construct the ACD model for block trading volume duration. The ACD model based on the block trading volume duration is referred to as volume ACD (VACD) in this study. The VACD model of this study will be tested against the higher-order of volume duration and higher-order of conditional mean volume duration to find the optimal order of VACD model. Then, the VACD model will be integrated into GARCH-type models. By integrating with GARCH-type models, the VACD-GARCH type models employed in this study, including VACD-GARCH, VACD-IGARCH and VACD-FIGARCH models, are applied to capture volume duration clustering. Finally, this study further tests the goodness of fit and assesses relative performance of prediction for these block trading volume and volatility models.

\section{Literature Review}

Kraus and Stoll (1972) suggest that the institutional investors gain from large amount of transactions which are regarded as short-term influenced factors of stock price through private information. For further research, the trading sizes can be imposed in modelling the relationships between price and volume, which are correlated with the private information about the true value of stock. However, the advantage of private information is captured by investors who would like to trade large quantities. A rational market maker will explain the large abnormal orders as evidence of trading by informed trader and will adjust beliefs and prices accordingly (Easley \& O'Hara, 1987).

Moreover, Gallant, Rossi, and Tauchen (1992) employ semi-nonparametric method to examine the joint density of price change and volume. Their empirical results also reveal that there is positive relationship between volume and volatility. If the market microstructure data are adopted in empirical analysis, the event of block trading volume can be viewed as driving both abnormal returns and volume processes. The empirical results reveal that the arrival of large trading volume event will produce significant abnormal returns (Aitken \& Frino, 2005; Shome \& Singh, 1995). Thus, block trading volume can be viewed as an important explanatory variable in the stochastic process of stock price.

Engle (1982) proposes the success of standard linear time series model, ARCH model, arising from the use of the conditional and unconditional variances. Then, Bollerslev (1986) proposes the generalized ARCH (GA
$\mathrm{RCH})$ model. Moreover, Lumsdaine (1992) indicates th at theoretical results appear to be no discontinuity in $t$ he finite sample distribution of the estimators in the I GARCH model. Baillie, Bollerslev and Mikkelsen (199 6) rely on an ARFIMA type model to derive FIGARC $\mathrm{H}$ model in representative to better capture the long $\mathrm{m}$ emory dynamic dependencies in the conditional varianc e. The FIGARCH model may be seen as natural exten sion of the IGARCH model, allowing for fractional or ders of integration in the autoregressive polynomial of the corresponding ARMA representation.

Heynen and Kat (1994) employ GARCH, EGARCH and stochastic volatility model to compare the performance between short-term and long-term forecasting. Heynen and Kat indicate that stochastic volatility model has better forecasting in stock index, but GARCH model has better forecasting in exchange rate. Yu (2000) employs stochastic volatility (SV) model to calculate forecasting errors from variances instead of from standard deviation. In addition, Mendes and Kolev (2008) investigate the interdependence in emerging markets, which can be driven by conditional short- and long-range dependence in volatility. Mendes and Kolev also fit copulas model to pairs of filtered returns, which using FIGARCH process to the joint excess residuals. Their empirical results indicate that long memory in volatility was responsible for chances in increasing external dependence.

Many studies are plausible and even arbitrary to analyze the time series data with econometric techniques of fixed time interval. However, there might be a significant loss of information by adopting this modelling framework. Transaction data arrive at irregular times occurring between two trades. The transaction information may be informative about the behavior of the traders. To resolve this problem, Engle and Russell (1998) introduce the autoregressive conditional duration (ACD) model, which captures more information between the arrival times of trades. The ACD model shares many similar features with the GARCH model (Engle, 2000: I, 2015; Go \& Lau, 2016; Jati \& Premaratne, 2017; Nguyen \& Nguyen, 2019).

Furthermore, the choice of the distribution of the error term impacts on the conditional intensity or hazard function of the ACD model. Since the volume can take only positive values, the support of the distribution of the error terms is positive real line. In addition, the GARCH-type models are not able to appropriately explain various findings of long memory phenomenon in the volatility of financial instruments. The general formulation of GARCH model for irregularly spaced financial data is extremely complex. Thus, Engle (2000) proposes the ACD-GARCH model to capture the volatilities of the asset returns. An ACDGARCH model is a random coefficient GARCH model, or doubly stochastic GARCH, where the duration between 
transactions may determine dynamics of the entire parameter vector. Based on this framework, this study tends to find more appropriate time series model to capture the volatility dynamics of financial instrument returns.

Suppose that the ACD effects are not appropriately captured by the estimated model, and some ACD effects are still present in the disturbances. A more generalized LM tests of the ACD models are proposed by Meitz and Terasvirta (2006), including tests against higher-order of ACD models and tests against no remaining ACD effects in the standardized duration. Moreover, Racicot, Théoret and Coen (2008) employ GARCH model, ACD-GARCH model, extended ACD-GARCH model and ultra-high-frequency GARCH (UHF-GARCH) models to forecast volatility. Their empirical results indicate that the ACD-GARCH model has not performed better than the realized volatility. $\mathrm{Ng}$ (2008) tends to apply the concept of large trading volume as an important explanatory variable in the stochastic process of stock price to capture the changing cluster structure of duration processes by analyzing the dynamics of volume duration with increasing threshold values. His empirical results indicate that the market's absorption limit for high volumes of shares, inducing additional time costs of liquidity when trading these quantities.

\section{Methodology}

The primary purpose of this study is to utilize the market microstructure data to examine the VACD based GARCHtype models of volatility of financial or economic time series.

\subsection{GARCH-type Model}

Autoregressive Conditional Heteroscedasticity (ARCH) model is proposed by Engle (1982), and is used for modelling and forecasting volatility. The $\mathrm{ARCH}(\mathrm{q})$ model can be written as follows:

$\varepsilon_{t}=v_{t} \sigma_{t} v_{t} \sim N(0,1)$

$\sigma_{t}^{2}=\alpha_{0}+\sum_{i=1}^{q} \alpha_{i} \varepsilon_{t-i}^{2}$

where $\alpha_{i} \geq 0, i=1,2, \cdots, q$, and $v_{t}$ is a Gaussian white noise process. The real-valued stochastic process is covariance stationary if and only if sum of the positive autoregressive parameters is less than one.

In empirical applications of ARCH(q) models, to avoid the problem of a long lag length and a large number of parameters, Bollerslev (1986) proposes the generalized ARCH (GARCH) model. The GARCH (p, q) model can be written as follows:

$\sigma_{t}^{2}=\omega+\alpha(L) \varepsilon_{t}^{2}+\beta(L) \sigma_{t}^{2}$

where $L$ denotes the lag operator, and $\alpha(L) \equiv \sum_{i=1}^{q} \alpha_{i} L^{i}$ and $\beta(L) \equiv \sum_{j=1}^{p} \beta_{j} L^{j}$. The conditional variances depend on the past q squared error terms and the past $\mathrm{p}$ conditional variances. For the conditional variance in $\operatorname{GARCH}(p, q)$ model to be well defined, all the coefficients in the corresponding infinite order linear ARCH model must be positive. Moreover, GARCH model is a more parsimonious model than ARCH model.

The ARCH model and GARCH model are applied to the time series data. Engle (1982) proposes the ARCH-LM (Lagrange Multiplier) test to examine ARCH effect. If the data are homoscedastic, then the variance cannot be predicted and variations in $\varepsilon_{t}^{2}$ will be purely random. If there exists ARCH effects, the large values of errors $\varepsilon_{t}^{2}$ can be predicted by past squared residuals. Thus, if the value of the test statistic is greater than the critical value from the $\chi^{2}$ distribution, then reject the null hypothesis. Moreover, Ljung and Box (1978) argue an asymptotically equivalent test for error term $\varepsilon_{t}^{2}$. If the null hypothesis can not be rejected, the error term follows white noise. However, if the test statistic rejects the null hypothesis, the error term still exhibits autocorrelation.

Following the $\operatorname{GARCH}(1,1)$ model for conditional normal errors, the available Monte Carlo evidence suggests that the estimate for $\alpha_{1}+\beta_{1}$ is downward biased and skewed to the right in small samples. The stationarity of this process is achieved directly when the following restriction is satisfied: $\alpha_{1}+\beta_{1}<1$. Engle and Bollerslev (1986) defined the $\operatorname{IGARCH}(\mathrm{p}, \mathrm{q})$ process:

$\varphi(L)(1-L) \varepsilon_{t}^{2}=\omega+[1-\beta(L)] u_{t}$

where $\varphi(L) \equiv[1-\alpha(L)-\beta(L)](1-L)^{-1}$ is of order m1 and $m \equiv \max \{p, q\}$, and $u_{t}=\varepsilon_{t}^{2}-\sigma_{t}^{2}$. Lumsdaine (1992) indicates that the theoretical results appear to be no discontinuity in the finite sample distribution of the estimators at the $\operatorname{IGARCH}(1,1)$ boundary, i.e. $\alpha_{1}+\beta_{1}=1$. The process of IGARCH allows the integration coefficient to vary in the interval of $[0,1]$.

According to Baillie, Bollerslev and Mikkenlsen (1996), a FIGARCH ( $, d, q)$ model for the conditional variance $\sigma_{t}^{2}$ satisfies

$\varphi(L)(1-L)^{d} \varepsilon_{t}^{2}=\omega+[1-\beta(L)] u_{t}$

where the fractional integration parameter $d \in[0,1]$. Baillie, Bollerslev, and Mikkenlsen (1996) claim that FIGARCH (p, d, q) process is ergodic and strictly stationary. 


\subsection{ACD Model}

Engle and Russell (1998) investigate the transaction data to generate expected long durations that lead to long durations, and short durations arrive at short durations. The property of volatilities of the market is analogous to the conditional variance of ARCH model. Thus, based on the previous framework of ARCH model, Engle and Russell (1998) propose the autoregressive conditional duration (ACD) models to assess the problem of irregular duration. The feature of transactions data is a vector observed at the time of the truncation that can identify or further describe the occurred event by Engle and Russell.

Let $t_{i}$ be the time at which the $i$-th trade occurs and let $x_{i} \equiv t_{i}-t_{i-1}$ denote the duration between block trading trades. At the $i$-th event the marks are observed and be denoted $y_{i}$, which is a $n \times 1$ vector from a sample space $\Omega$. The time dependence can be summarized by a function $\psi$ which is the conditional mean duration given past information and has the feature that $x_{i} / \psi_{i}$ is independent and identically distributed. The density of standardized duration can be depicted as follows:

$$
\begin{aligned}
& E\left(x_{i} \mid x_{i-1}, x_{i-2}, \cdots, x_{1}\right)=\psi\left(x_{i} \mid x_{i-1}, x_{i-2}, \cdots, x_{1}, \theta\right) \equiv \\
& \psi_{i}, \quad x_{i} / \psi_{i} \text { are i.i.d. }
\end{aligned}
$$

In equation (6), autoregressive conditional duration model parameterizes the conditional duration in terms of the lagged duration. Equation (6) can be written as

$x_{i}=\psi_{i} e_{i}$

where $e_{i}$ follows i.i.d. series of distribution which must be specified. The probability density function of equation (7) is $p_{i}(e ; \delta)$ and $\delta$ is a parameter vector.

The hazard function $\lambda_{0}(t)$ is often called the baseline hazard function since it does not depend upon conditional information:

$\lambda_{0}(t)=\frac{P_{0}(t)}{S_{0}(t)}$

where $P_{0}$ is a probability distribution of error term; $S_{0}$ is a survival function of error function. The generalized probability density of ACD model can be expressed as:

$\lambda\left(t \mid N(t), \mathrm{t}_{1}, \ldots, \mathrm{t}_{N(t)}\right)=\lambda_{0}\left(\frac{\mathrm{t}-\mathrm{t}_{N(t)}}{\psi_{N(t)+1}}\right) \frac{1}{\psi_{N(t)+1}}$

Equation (9) reveals that the past marked information through cumulated effect and moving average would impact the baseline hazard function. The probability density function can be expressed as follows:

$$
\lambda\left(t \mid N(t), \mathrm{t}_{1}, \ldots, \mathrm{t}_{N(t)}\right)=\frac{1}{\psi_{N(t)+1}}
$$

The generalized model derives infinite memory by mmemory of the intensity and lagged $\mathrm{n}$ durations, which is called the $\operatorname{ACD}(\mathrm{m}, \mathrm{n})$ model. The $i$-th conditional mean of $x_{i}$ is determined simultaneously by the $m$ durations and the past $n$ conditional means of duration:

$\psi_{i}=\omega+\sum_{\mathrm{j}=1}^{m} \alpha_{j} x_{\mathrm{i}-\mathrm{j}}+\sum_{\mathrm{j}=1}^{n} \beta_{j} \psi_{\mathrm{i}-\mathrm{j}}$

where the constraints consist of $\omega>0 ; \alpha=\left(\alpha_{1}, \alpha_{2}, \cdots \alpha_{m}\right) \geq 0 ; \beta=\left(\beta_{1}, \beta_{2}, \cdots \beta_{n}\right) \geq 0$; $\alpha+\beta<1$ to assure that the stochastic process reaches the stationarity property.

The ACD model shares many similar features with the GARCH model. However, the difference between two models is that GARCH model is used to predict the volatility clustering, while ACD model is used to portray the clustering duration (Engle, 2000). The marginal density of the block trading volume duration on past block trading volume duration is the conditional density and conditional on past volume duration.

It is relatively difficult determining the optimal order (m, n) of $m$ durations and $n$ conditional mean durations to apply ACD model in empirical analysis. To determine the optimal order of the ACD (m, n) model, Meitz and Terasvirta (2006) propose generalized LM tests, including testing against higher-order models of ACD and no remaining ACD effects in the standardized duration. This study imposes these LM tests to examine the VACD model and to determine the optimal lagged order of VACD model.

\subsection{Testing Higher-Order VACD Model}

This study employs the misspecification proposed by Meitz and Terasvirta (2006). The process of testing the $\operatorname{VACD}(\mathrm{m}, \mathrm{n})$ against the higher-order $\operatorname{VACD}(\mathrm{m}+\mathrm{r}, \mathrm{n})$ is governed by

$$
\begin{gathered}
x_{i}=\left(\psi_{i}+\phi_{i}\right) e_{i} \\
\psi_{i}+\phi_{i}=\omega+\sum_{j=1}^{m+r} \alpha_{j} x_{i-j}+\sum_{j=1}^{n} \beta_{j}\left(\psi_{i-j}+\phi_{i-j}\right) \\
\phi_{i}=\sum_{j=m+1}^{m+r} \alpha_{j} x_{i-j}+\sum_{j=1}^{n} \beta_{j} \phi_{i-j} \quad e_{i} \sim \text { i. i.dexp }(1)
\end{gathered}
$$

The null hypothesis is $H_{0}: \phi_{i} \equiv 0$, i.e., $\alpha_{m+1}=\cdots=$ $\alpha_{m+r}=0$. If the null hypothesis that lower-order model is correctly specified can not be rejected, the model collapses to VACD (m, n) model. If the null hypothesis is rejected, then $\operatorname{VACD}(\mathrm{m}, \mathrm{n})$ of higher-order $(m+r)$ durations and $n$ conditional mean durations is required.

Meitz and Terasvirta (2006) propose Lagrange Multiplier 
(LM) test to evaluate VACD model. Let $\quad \theta_{1}=\left(\omega, \alpha_{1}, \ldots, \alpha_{m}, \beta_{1}, \ldots, \beta_{n}\right)^{\prime} \quad$ and $\theta_{2}=\left(\alpha_{m+1}, \ldots, \alpha_{m+r}\right)^{\prime}$. The procedure of additively misspecified test can be specified as the null hypothesis $H_{0}: \theta_{2}=0$. The quasi maximum likelihood (QML) estimate of $\theta_{1}$ is obtained under the null hypothesis. Then, the test statistics of the LM test can be computed as follows:

$$
\begin{aligned}
& L M= \\
& \left\{\sum_{i=1}^{k} \hat{c}_{i} \hat{b}_{i}^{\prime}\right\} \times \\
& \left\{\sum_{i=1}^{k} \hat{b}_{i} \hat{b}_{i}^{\prime}-\left(\sum_{i=1}^{k} \hat{b}_{i} \hat{a}_{i}^{\prime}\right)\left(\sum_{i=1}^{k} \hat{a}_{i} \hat{a}_{i}^{\prime}\right)^{-1}\left(\sum_{i=1}^{k} \hat{a}_{i} \hat{b}_{i}^{\prime}\right)\right\}^{-1} \times \\
& \left\{\sum_{i=1}^{k} \hat{c}_{i} \hat{b}_{i}\right\}(13)
\end{aligned}
$$

where

$$
\begin{gathered}
\hat{a}_{i}=\frac{1}{\hat{\psi}_{i}} \frac{\partial \hat{\psi}_{i}}{\partial \theta_{1}}=\hat{\psi}_{i}^{-1}\left(1, x_{i-1}, \ldots, x_{i-m}, \hat{\psi}_{i-1}, \ldots, \hat{\psi}_{i-n}\right)^{\prime} \\
+\hat{\psi}_{i}^{-1} \sum_{j=1}^{n} \hat{\beta}_{j} \frac{\partial \hat{\psi}_{i-j}}{\partial \theta_{1}} ; \\
\hat{b}_{i}=\frac{1}{\hat{\psi}_{i}} \frac{\partial \hat{\phi}_{i}}{\partial \theta_{2}}=\hat{\psi}_{i}^{-1}\left(x_{i-m-1}, \ldots, x_{i-m-r}\right)^{\prime} \\
+\hat{\psi}_{i}^{-1} \sum_{j=1}^{n} \hat{\beta}_{j} \frac{\partial \hat{\phi}_{i-j}}{\partial \theta_{2}} ; \\
\hat{c}_{i}=\frac{x_{i}}{\psi_{i}\left(\hat{\theta}_{1}\right)}-1 \quad i=1,2, \ldots, k
\end{gathered}
$$

The LM test statistics have an asymptotic $\chi^{2}$ distribution with $\operatorname{dim}\left(\theta_{2}\right)$ degrees of freedom.

The process of testing the VACD $(\mathrm{m}, \mathrm{n})$ against higherorder VACD $(m, n+r)$ is governed by

$$
\begin{gathered}
x_{i}=\left(\psi_{i}+\phi_{i}\right) e_{i} \\
\psi_{i}+\phi_{i}=\omega+\sum_{j=1}^{m} \alpha_{j} x_{i-j}+\sum_{j=1}^{n+r} \beta_{j}\left(\psi_{i-j}+\phi_{i-j}\right) \\
\phi_{i}=\sum_{j=n+1}^{n+r} \beta_{j}\left(\psi_{i-j}+\phi_{i-j}\right)+\sum_{j=1}^{n} \beta_{j} \phi_{i-j} \\
e_{i} \sim \text { i.i.d. } \exp (1)
\end{gathered}
$$

The null hypothesis is $H_{0}: \phi_{i} \equiv 0$, i.e., $\beta_{n+1}=\cdots=$ $\beta_{n+r}=0$. If the null hypothesis that the model of order $m$ and $n$ are correctly specified can not be rejected, the model collapses to $\operatorname{ACD}(m, n)$ model. If the null hypothesis is rejected, then $\operatorname{VACD}(m, n)$ of higher-order $m$ durations and $(n+r)$ conditional mean durations is required.

Let $\theta_{1}=\left(\omega, \alpha_{1}, \ldots, \alpha_{m}, \beta_{1}, \ldots, \beta_{n}\right)^{\prime}$ and $\theta_{2}=$ $\left(\beta_{n+1}, \ldots, \beta_{n+r}\right)^{\prime}$. The procedure of additively misspecified LM test can be expressed as the null hypothesis $H_{0}: \theta_{2}=0$. The quasi maximum likelihood estimate of is estimated, and the test statistics are computed as follows:

$$
\begin{aligned}
& \hat{a}_{i}=\frac{1}{\hat{\psi}_{i}} \frac{\partial \hat{\psi}_{i}}{\partial \theta_{1}}=\hat{\psi}_{i}^{-1}\left(1, x_{i-1}, \ldots, x_{i-m}, \hat{\psi}_{i-1}, \ldots, \widehat{\psi}_{i-n}\right)^{\prime} \\
& +\hat{\psi}_{i}^{-1} \sum_{j=1}^{n} \hat{\beta}_{j} \frac{\partial \hat{\psi}_{i-j}}{\partial \theta_{1}} ; \\
& \hat{b}_{i}=\frac{1}{\hat{\psi}_{i}} \frac{\partial \hat{\phi}_{i}}{\partial \theta_{2}}=\hat{\psi}_{i}^{-1}\left(\hat{\psi}_{i-n-1}, \ldots, \hat{\psi}_{i-n-r}\right)^{\prime} \\
& +\widehat{\psi}_{i}^{-1} \sum_{j=1}^{n} \hat{\beta}_{j} \frac{\partial \hat{\phi}_{i-j}}{\partial \theta_{2}} \\
& \hat{c_{i}}=\frac{x_{i}}{\psi_{i}\left(\hat{\theta}_{1}\right)}-1 \quad i=1,2, \ldots, k \text { 。 }
\end{aligned}
$$

The LM test statistic has an asymptotic $\chi^{2}$ distribution with $\operatorname{dim}\left(\theta_{2}\right)$ degrees of freedom.

\subsection{VACD-FIGARCH Model}

The block trading volume duration based ACD model will be referred to as Volume ACD (VACD) in this study. To capture the long memory of volatility in the event of block trading, this study employs VACD-FIGARCH model of the VACD-GARCH framework.

The general formulation of GARCH for irregularly spaced financial data is extremely complex. Thus, Engle (2000) proposes employing ACD-GARCH model to capture the past asset return volatilities. An ACD-GARCH model is in fact a random coefficient GARCH model, or doubly stochastic GARCH, where the duration between transactions determine dynamics of the entire parameter vector.

Engle (2000) proposes the generalized non-linear model to examine the volatility of price return within transaction information. The prices convey information about the volatility of the market and a variety of market microstructure data. This study imposes the ultra-highfrequency GARCH model by Engle (2000). Let the return from block trading transaction $i-1$ to $i$ be $r_{i}$, the conditional variance pre transaction is defined as

$V_{i-1}\left(r_{i} \mid x_{i}\right)=h_{i}$

where $h_{i}$ indicates that the variance is conditioned on the current duration as well as the past returns and duration. The conditional volatility per unit of time is defined as

$V_{i-1}\left(\frac{r_{i}}{\sqrt{x_{i}}} \mid x_{i}\right)=\sigma_{i}^{2}$

The conditional variance can be rewritten as follows:

$h_{i}=x_{i} \sigma_{i}^{2}$ 
The expected transaction variance conditional on past block trading volume duration are given by

$E_{i-1}\left(h_{i}\right)=E_{i-1}\left(x_{i} \sigma_{i}^{2}\right)$

A conventional $\operatorname{GARCH}(\mathrm{p}, \mathrm{q})$, i.e., $\sum_{i=1}^{q} \alpha_{i} \varepsilon_{t-i}^{2}+\sum_{i=1}^{p} \beta_{i} \sigma_{t-i}^{2}$, can be estimated with the dependent variable defined as returns divided by the square root of the time, $r / \sqrt{x}$. The $\operatorname{GARCH}(\mathrm{p}, \mathrm{q})$ can be further extended into ACD-GARCH model. That is,

$\sigma_{t}^{2}=\omega+\sum_{i=1}^{q} \alpha_{i} \varepsilon_{t-i}^{2}+\sum_{i=1}^{p} \beta_{j} \sigma_{t-j}^{2}+\gamma x_{t}^{-1}$

where $x_{t}^{-1}$ is the reciprocal of duration, which is used to examine the relationship between the block trading volume duration and volatility.

The VACD-GARCH model employed in this study is based on volume duration. Moreover, this study also incorporates VACD into the IGARCH model and FIGARCH model to construct VACD $(\mathrm{m}, \mathrm{n})-\operatorname{IGARCH}(\mathrm{p}, \mathrm{q})$ and VACD $(\mathrm{m}, \mathrm{n})$-FIGARCH $(\mathrm{p}, \mathrm{d}, \mathrm{q})$, respectively. Thus, a more realistic specification for the conditional variance is as follows:

$\varphi(L)(1-L) \varepsilon_{t}^{2}=\omega+[1-\beta(L)] u_{t}+\gamma x_{t}^{-1}$

$\varphi(L)(1-L)^{d} \varepsilon_{t}^{2}=\omega+[1-\beta(L)] u_{t}+\gamma x_{t}^{-1}$

The above equations allow the conditional variance to depend upon the reciprocal of volume duration. The $\gamma$ coefficient can be tested under Easley and O'Hara (1992). They indicate that the longer the volume duration, the smaller the volatility of stock price and vice versa.

Differing from many previous studies, this study utilizes the market microstructure data of block trading volume duration and incorporates it into volatility models. Then, this study employs the VACD-GARCH framework to capture the long memory of volatility in event of block trading by employing VACD-FIGARCH model. The density of the disturbance is specified to be exponential ACD model. Moreover, this study adopts the LM test to examine the block trading volume duration with an optimal lagged order VACD $\left(m^{*}, n^{*}\right)$ model. The result of error term from VACD $\left(\mathrm{m}^{*}, \mathrm{n}^{*}\right)$ model are imposed into the FIGARCH model, i.e., $\operatorname{VACD}\left(\mathrm{m}^{*}, \mathrm{n}^{*}\right)$-FIGARCH $(\mathrm{p}, \mathrm{d}, \mathrm{q})$, to capture the volatility process. In order to maintain the quality of estimated model and to avoid over fitting, this study employs AIC (Akaike's information criterion; Akaike, 1976) to determine the appropriate goodness of fit of the estimated models. The lower value the information criterion is, the better the goodness of fit.

This study employs in-sample data and the estimated parameters to forecast the volatility of VACD-GARCH type models and to compare the relative prediction performance of the relevant models. To assess the relative predication performance, this study employs the criteria of mean absolute error (MAE) and root mean square error (RMSE).

\section{Empirical Results and Analysis}

This study will first explain the source of volume duration data and then empirically investigate the dependence structure of the duration clustering of the block trading volume.

\subsection{Data Description}

This study takes into account the liquidity of stock, and selects Chunghwa Telecom (CHT) Inc., offering the America Depository Receipt (ADR) in NYSE, to investigate the duration clustering of the block trading volume in Taiwanese equity market. Moreover, the regulations of Taiwanese equity market exhibit that each quote of the block trading of a single security will be limited to 500 or more trading units of a listed security. The quote of the block trading will be separated into different quotes in clustering time. The single transaction volume does not represent block trading. The limitation of this study is that it uses the cumulated volume to determine block trading. Therefore, this study imposes each 500 or more cumulated units of a listed security as block trading volume, and the volume duration is computed between two block trading volumes of time. Apart from the closing auction, the trading time of Taiwanese equity market is continuous from 9:00 to 13:25. The settlement regulations of Taiwanese equity market adopt the 5-minute collective auction at closing. Thus, this study eliminates last fiveminute trading data from the sample of this study.

This study employs cubic spline, proposed by Engle and Russell (1998), to examine the diurnally adjusted volume duration. The trading time is separated into several intervals. Each interval represents thirty minutes. The mean volume duration is calculated according to its corresponding time interval. The diurnally adjusted factor is given by

$\varphi\left(t_{i}\right)=\sum_{\mathrm{j}=1}^{9} I_{j}\left[c_{j}+d_{1 j}\left(t_{i}-k_{j}\right)+d_{2 j}\left(t_{i}-k_{j}\right)^{2}+\right.$ $\left.d_{3 j}\left(t_{i}-k_{j}\right)^{3}\right]$

with diurnally adjusted factor $\varphi\left(t_{i}\right)$; the $j$-th time indicator $I_{j}$, and $I_{j}=1$ when $k_{j} \leq t_{i}<k_{j+1}$; the mean volume duration $c_{j}$ of $j$-th time interval; estimated parameters $d_{1 j}, d_{2 j}$ and $d_{3 j}$; each cumulated volume $t_{i}$, and each time interval $k_{j}$ of initial time. The raw volume duration is adjusted by diurnal factor and can be written as 
$\tilde{x}_{i}=\frac{x_{i}}{\varphi\left(t_{i}\right)}$

where the de-seasonalized volume duration $\tilde{x}_{i}$ should not exhibit diurnal pattern.

The descriptive statistics of raw and adjusted volume duration are given in Table1. The data set analyzed consists of volume duration of CHT Inc. in Taiwanese equity market from January 2, 2008 through Nonmember 28, 2008 for a total of 12978 observations. It can be seen from Table 1 that the average raw and adjusted volume durations are 146.823 and .998 seconds with a variance of 7180.794 and .326 , respectively. The daily patterns of raw and adjusted volume duration are plotted in Figure 1. The inverted $U$ shape of raw volume duration in Figure 1 indicates that the opening and closing trading time are very active with block trading occurring, and the middle of the day tends to exhibit longer volume duration. It also is indicated in Figure 1 that the adjusted volume duration exhibits the daily seasonal factor, which can be approximated by cubic spline.

\subsection{Empirical Results and Analysis}

The ADF and PP unit root tests are conducted to verify the stationarity of stock returns of CHT Inc.. The results indicate that the series of volume duration is stationary and the series contains an ARCH-effect.

It is assumed in this study that the error term of ACD model follows the exponential distribution. The corresponding exponential ACD model employs quasi maximum likelihood function to estimate the parameters.

Table 1: The Descriptive Statistics of Raw and Adjusted Volume Duration

\begin{tabular}{|c|c|c|}
\hline & Volume Duration & $\begin{array}{c}\text { Adjusted Volume } \\
\text { Duration }\end{array}$ \\
\hline Sample & 12978 & 12978 \\
\hline Minimum & 25.300 & 0.152 \\
\hline Maximum & 350.520 & 3.081 \\
\hline Mean & 146.823 & 0.998 \\
\hline Variance & 7180.794 & 0.326 \\
\hline Skewness & 0.561 & 0.587 \\
\hline Kurtosis & 2.357 & 2.523 \\
\hline & $\mathrm{ACF}$ & -0.002 \\
\hline lag 1 & 0.019 & 0.008 \\
\hline lag 6 & 0.028 & 0.004 \\
\hline lag 12 & 0.025 & 0.009 \\
\hline lag 18 & 0.030 & \\
\hline \multicolumn{3}{|c|}{ PACF } \\
\hline
\end{tabular}

\begin{tabular}{|c|c|c|}
\hline $\operatorname{lag} 1$ & 0.019 & -0.002 \\
\hline $\operatorname{lag} 6$ & 0.026 & 0.008 \\
\hline $\operatorname{lag} 12$ & 0.022 & 0.004 \\
\hline $\operatorname{lag} 18$ & 0.026 & 0.008 \\
\hline Ljung-Box (18) & $149.378^{* * *}$ & $113.133^{* * *}$ \\
\hline & $(0.000)$ & $(0.000)$ \\
\hline
\end{tabular}

Note: 1 . The unit of volume duration is second.

2. *** represents significant at the $1 \%$ level of significance.
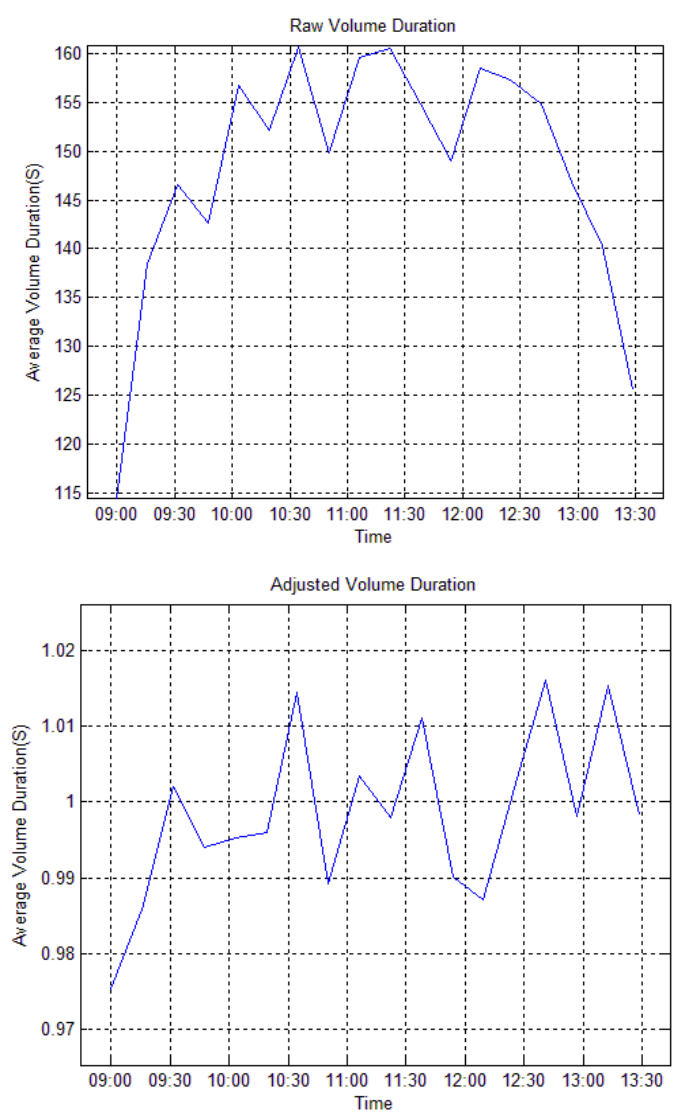

Figure 1: The Daily Pattern of Raw and Adjusted Volume Duration

The log likelihood function of exponential ACD model can be written as

$L L F=\sum_{i=1}^{N(T)} \ln \left(\frac{1}{x_{i}}\right)+\ln \left(\frac{\Gamma(2) x_{i}}{\psi_{i}}\right)-\left(\frac{\Gamma(2) x_{i}}{\psi_{i}}\right)$

The estimated parameters of volume duration for the exponential ACD are presented in Table 2. As indicated in Table 2, the parameters of conditional mean volume duration are all significant at $1 \%$ level of significance. The sum of $\alpha_{i}$ and $\beta_{i}$ of CHT Inc. volume duration of ACD model is less than one, indicating that it is a stationary process. 
The statistics of conditional mean volume duration are presented in Table 3. The empirical results reveal in Table 3 that the mean of conditional mean volume duration is asymptotic to one, which matches the constraint of positive conditional mean volume duration.

The tests conducted for the VACD model of volume duration of CHT Inc. are presented in Table 4. Specifically, the results of the LM tests of $\operatorname{VACD}(m, n)$ against the higher-order of volume duration $\operatorname{VACD}(m+1, n)$ and the higher-order of conditional mean volume duration VACD $(\mathrm{m}, \mathrm{n}+1)$, and against no remaining VACD are presented in Table 4.

If the null hypothesis of LM test of VACD is rejected, the VACD should be fitted higher-order of the lagged duration or lagged conditional mean duration. The null hypothesis of LM test of VACD $(1,1)$ model against the higher-order of volume duration VACD $(2,1)$ can not be rejected. Nevertheless, the null hypothesis of LM test of VACD $(1,1)$ model against the higher-order of conditional mean volume duration VACD $(1,2)$ is rejected. Thus, VACD $(1,1)$ model is not appropriate to capture volume duration clustering. The order of the VACD $(1,1)$ model is too low for the specification of the volume duration clustering. Therefore, the consecutive higher-order duration of VACD model, e.g. $\operatorname{VACD}(1,2)$ or VACD $(2,1)$, is needed to fit and portray the volume duration clustering.

Table 2: The Estimated Parameters of Volume Duration $\psi_{i}=\omega+\sum_{j=1}^{m} \alpha_{j} x_{i-j}+\sum_{j=1}^{n} \beta_{j} \psi_{i-j} m=1,2,3 \quad n=1,2,3$

\begin{tabular}{|c|c|c|c|c|}
\hline & VACD (1,1) & VACD (1,2) & VACD (1,3) & VACD (2,1) \\
\hline$\omega$ & $0.132 * * *$ & $0.349^{* * *}$ & $0.367^{* * *}$ & $0.407^{* * *}$ \\
\hline & $(0.000)$ & $(0.000)$ & $(0.000)$ & $(0.000)$ \\
\hline$\alpha_{1}$ & 0.001 & 0.002 & -0.001 & -0.002 \\
\hline & 0.926 & 0.899 & 0.930 & 0.883 \\
\hline$\alpha_{2}$ & & & & 0.007 \\
\hline & & & & 0.626 \\
\hline$\alpha_{3}$ & & & & \\
\hline & & & & $0.587 * * *$ \\
\hline$\beta_{1}$ & $0.867 * * *$ & $0.454^{* * *}$ & $0.205^{* * *}$ & $(0.000)$ \\
\hline & $(0.000)$ & $(0.000)$ & $(0.000)$ & \\
\hline$\beta_{2}$ & & $0.195 * * *$ & $0.152^{* * *}$ & \\
\hline & & $(0.000)$ & $(0.000)$ & \\
\hline$\beta_{3}$ & & & $0.276^{* * *}$ & \\
\hline & & & $(0.000)$ & \\
\hline
\end{tabular}

Note: $1 .{ }^{* * *}$ represents significant at the $1 \%$ level of significance.

To test whether $\operatorname{VACD}(1,2)$ is appropriate to capture volume duration clustering, the null hypotheses of VACD $(1,2)$ against $\operatorname{VACD}(2,2)$ and $\operatorname{VACD}(1,3)$ are conducted in empirical analysis. The null hypothesis of LM test of $\operatorname{VACD}(1,2)$ against VACD $(1,3)$ also is rejected. However, the null hypothesis of LM test of $\operatorname{VACD}(1,2)$ against VACD $(2,2)$ can not be rejected. Thus, the order of the VACD $(1,2)$ also is too low for the specification of the volume duration clustering. Therefore, the higher-order of VACD, e.g. VACD $(1,3)$ or VACD $(2,2)$, is required to fit and depict the volume duration clustering.

To test whether VACD $(2,1)$ is appropriate to capture volume duration clustering, the null hypotheses of VACD $(2,1)$ against $\operatorname{VACD}(3,1)$ and $\operatorname{VACD}(2,2)$ are conducted in empirical analysis. The null hypothesis of LM test of VACD $(2,1)$ against VACD $(2,2)$ also is rejected. Nevertheless, the null hypothesis of LM test of VACD $(2,1)$ against VACD $(3,1)$ can not be rejected. The order of the $\operatorname{VACD}(2,1)$ model also is too low for the specification of the volume duration clustering. Therefore, the higher-order of VACD model, e.g. VACD $(2,2)$ or $\operatorname{VACD}(3,1)$, is required to fit and depict the volume duration clustering.

Table 3: Ctd

\begin{tabular}{|c|c|c|c|c|}
\hline & VACD (2,2) & VACD (2,3) & VACD (3,1) & VACD (3,2) \\
\hline$\omega$ & $0.386^{* * *}$ & $0.158^{* * *}$ & $0.696^{* * *}$ & $0.200^{* * *}$ \\
\hline & $(0.000)$ & $(0.000)$ & $(0.000)$ & $(0.000)$ \\
\hline$\alpha_{1}$ & $(0.000)$ & 0.002 & -0.002 & -0.003 \\
\hline & 0.977 & 0.902 & 0.799 & 0.847 \\
\hline$\alpha_{2}$ & 0.004 & $(0.000)$ & 0.001 & -0.001 \\
\hline & 0.772 & 0.999 & 0.923 & 0.969 \\
\hline$\alpha_{3}$ & & & 0.013 & 0.006 \\
\hline & & & 0.191 & 0.712 \\
\hline$\beta_{1}$ & $0.403 * * *$ & $0.134 * * *$ & $0.290 * * *$ & $0.349 * * *$ \\
\hline & $(0.000)$ & $(0.000)$ & $(0.000)$ & $(0.000)$ \\
\hline$\beta_{2}$ & $0.206^{* * *}$ & $0.273^{* * *}$ & & $0.449 * * *$ \\
\hline & $(0.000)$ & $(0.000)$ & & $(0.000)$ \\
\hline$\beta_{3}$ & & $0.433^{* * *}$ & & \\
\hline & & $(0.000)$ & & \\
\hline
\end{tabular}

Note: $1 .{ }^{* * *}$ represents significant at the $1 \%$ level of significance.

The VACD $(m, n)$ model is consecutively tested against higher-order of volume duration model $\operatorname{VACD}(\mathrm{m}+1, \mathrm{n})$ and conditional mean volume duration model VACD (m, $n+1)$. Among all tests of null hypotheses of $\operatorname{VACD}(m, n)$ models, both the null hypotheses of VACD $(2,2)$ against higher-order of volume duration and conditional mean volume duration are rejected. However, at least one of the null hypotheses can not be rejected for the rest of null hypotheses of VACD $(\mathrm{m}, \mathrm{n})$ model. Therefore, to select the relatively more appropriate VACD model to be integrated into the GARCH type models, the case of $\operatorname{VACD}(2,2)$ 
model is eliminated as a candidate due to its rejection of both null hypotheses. One of the remaining VACD models will be chosen to be integrated into GARCH-type models. These $\operatorname{VACD}(\mathrm{m}, \mathrm{n})$ candidate models are selected based on the maximum value of LLF.

Table 4: The Descriptive Statistics of Conditional Mean Volume Duration

\begin{tabular}{|c|c|c|c|}
\hline & Min & Max & Mean \\
\hline $\operatorname{VACD}(1,1)$ & 0.995 & 1.003 & 0.999 \\
\hline $\operatorname{VACD}(1,2)$ & 0.995 & 1.003 & 0.998 \\
\hline $\operatorname{VACD}(1,3)$ & 0.995 & 1.001 & 0.999 \\
\hline $\operatorname{VACD}(2,1)$ & 0.986 & 1.018 & 0.998 \\
\hline $\operatorname{VACD}(2,2)$ & 0.991 & 1.011 & 0.998 \\
\hline $\operatorname{VACD}(2,3)$ & 0.994 & 1.005 & 0.999 \\
\hline $\operatorname{VACD}(3,1)$ & 0.981 & 1.030 & 0.998 \\
\hline $\operatorname{VACD}(3,2)$ & 0.988 & 1.013 & 0.999 \\
\hline
\end{tabular}

Table 5: The LM Tests of VACD Model of Volume Duration of CHT Inc

\begin{tabular}{|c|c|c|}
\hline & $\begin{array}{c}\text { VACD(m,n) } \\
\text { VS } \\
\text { VACD(m+1,n) }\end{array}$ & $\begin{array}{c}\text { VACD(m,n) } \\
\text { VS } \\
\text { VACD(m,n+1) }\end{array}$ \\
\hline $\operatorname{VACD~}(1,1)$ & 1.009 & $450590.000^{* * *}$ \\
\hline $\operatorname{VACD}(1,2)$ & 1.053 & $8050.400^{* * *}$ \\
\hline $\operatorname{VACD}(1,3)$ & 0.1323 & $53568.000^{* * *}$ \\
\hline $\operatorname{VACD}(2,1)$ & 2.224 & $7469.100^{* * *}$ \\
\hline $\operatorname{VACD}(2,2)$ & $14.551^{* * *}$ & $1022.200^{* * *}$ \\
\hline $\operatorname{VACD}(2,3)$ & 0.205 & $4056.000^{* * *}$ \\
\hline $\operatorname{VACD}(3,1)$ & 0.710 & $807.013^{* * *}$ \\
\hline $\operatorname{VACD}(3,2)$ & 0.500 & $187.150^{* * *}$ \\
\hline
\end{tabular}

Note: $1 .{ }^{* * *}$ represents significant at the $1 \%$ level of significance.

Based on the GARCH-type models in Table 4, the VACD $(2,1)$, which has the maximum value $-12,952.542$ of LLF, is relatively more appropriate to capture volume duration clustering. Thus, this study employs the $\operatorname{VACD}(2,1)$ model to be integrated into GARCH-type models and capture the property of the long-term memory in volatility.

The VACD based GARCH-type models employed in this study include VACD-GARCH, VACD-IGARCH and VACD-FIGARCH models, respectively. Firstly, this study employs the VACD $(2,1)$-GARCH $(m, n)$ model to capture the volume duration clustering. The estimated results of the VACD $(2,1)-G A R C H ~(m, n)$ models of CHT Inc. volume duration are presented in Table 5. As indicated in Table 5, all coefficients of the variable of the reciprocal of duration are significantly positive in $\operatorname{VACD}(2,1)-\operatorname{GARCH}(\mathrm{m}, \mathrm{n})$ candidate models. It indicates that the longer the volume duration, the smaller the volatility of stock price and vice versa. The significantly positive value of the coefficient of volume duration reveals that the volatility of stock price is affected by the volume duration of CHT Inc.. Moreover, this study employs information criterion of AIC to determine the goodness of fit of VACD based GARCH type models. Based on the AIC criteria, the VACD $(2,1)$ GARCH $(2,1)$ model exhibits the lowest value 0.3046 of AIC and is selected as the candidate model to be employed to forecast the volatility of stock returns of CHT Inc..

Next, this study employs the VACD $(2,1)-\operatorname{IGARCH}(m, n)$ model to depict the volume duration clustering. The estimation results of the $\operatorname{VACD}(2,1)-\operatorname{IGARCH}(\mathrm{m}, \mathrm{n})$ models of CHT Inc. volume duration are presented in Table 6. As indicated in Table 6, all estimated coefficients of the variable of the reciprocal of duration are significantly positive in VACD $(2,1)$-IGARCH $(\mathrm{m}, \mathrm{n})$ models, revealing that the volatility of stock price is affected by the volume duration of CHT Inc.. Based on the AIC criteria, the VACD $(2,1)-\operatorname{IGARCH}(1,1)$ model exhibits the lowest value 0.3130 of AIC and is selected as the candidate model to forecast the volatility of stock returns of CHT Inc.

Table 6: The Estimated Parameters of VACD-GARCH Model of Volume Duration

\begin{tabular}{|c|c|c|c|c|}
\hline & $\begin{array}{c}\text { VACD }(2,1)- \\
\text { GARCH } \\
(1,1)\end{array}$ & $\begin{array}{c}\text { VACD }(2,1)- \\
\text { GARCH } \\
(1,2)\end{array}$ & $\begin{array}{c}\text { VACD } \\
(2,1)- \\
\text { GARCH } \\
(2,1)\end{array}$ & $\begin{array}{c}\text { VACD } \\
(2,1)- \\
\text { GARCH } \\
(2,2)\end{array}$ \\
\hline \multirow[t]{2}{*}{$w$} & $0.0043 * * *$ & $\begin{array}{l}0.0043 \\
* * *\end{array}$ & $\begin{array}{l}0.0028 \\
* * *\end{array}$ & $0.0028 * * *$ \\
\hline & $(0.0000)$ & $(0.0000)$ & $(0.0000)$ & $(0.0000)$ \\
\hline \multirow[t]{2}{*}{$\alpha_{1}$} & $0.0054 * * *$ & $0.0054 * * *$ & $0.0034 * * *$ & $0.0034 * * *$ \\
\hline & $(0.0000)$ & $(0.0000)$ & $(0.0000)$ & $(0.0000)$ \\
\hline \multirow[t]{2}{*}{$\alpha_{2}$} & & $0.0012 * * *$ & & $0.0007 * * *$ \\
\hline & & $(0.0000)$ & & $(0.0000)$ \\
\hline \multirow[t]{2}{*}{$\beta_{1}$} & $0.9895 * * *$ & $0.9883 * * *$ & $0.5357 * * *$ & $0.5361 * * *$ \\
\hline & $(0.0000)$ & $(0.0000)$ & $(0.0000)$ & $(0.0000)$ \\
\hline \multirow[t]{2}{*}{$\beta_{2}$} & & & $0.4575 * * *$ & $0.4564 * * *$ \\
\hline & & & $(0.0000)$ & $(0.0000)$ \\
\hline \multirow[t]{2}{*}{$\gamma$} & $0.0027 * * *$ & $\begin{array}{l}0.0027 \\
* * *\end{array}$ & $0.0019 * * *$ & $0.0019 * * *$ \\
\hline & $(0.0000)$ & $(0.0000)$ & $(0.0000)$ & $(0.0000)$ \\
\hline AIC & 0.3136 & 0.3171 & 0.3046 & 0.3067 \\
\hline
\end{tabular}

Note: $1 .{ }^{* * *}$ represents significant at the $1 \%$ level of significance.

This study employs the VACD $(2,1)$-FIGARCH $(\mathrm{m}, \mathrm{d}, \mathrm{n})$ 
model to depict the volume duration clustering and long memory in volatility process. The estimated results of the VACD (2,1)-FIGARCH (m, d, n) models of CHT Inc. of volume duration are presented in Table 7. As indicated in Table 7, all estimated coefficients of the variables of the reciprocal of duration are significantly positive in VACD $(2,1)$-FIGARCH $(m, d, n)$ candidate models, indicating that the volatility of stock price is affected by the volume duration of CHT Inc.. The coefficient $d$ of the power of fractional integrated VACD (2,1)-FIGARCH $(\mathrm{m}, \mathrm{d}, \mathrm{n})$ models is significant at $1 \%$ level of significance. The estimated coefficients $d$ are lower than 0.5 , indicating that there is weak long memory dependence in volatility process. The long memory in volume duration series increases dependence at level of volatility clustering. The volume duration of interdependence can be an effect of long memory in volatility, and the long memory in volatility is corresponding for changing in dependence structure. Based on the AIC criteria, the VACD $(2,1)$-FIGARCH $(3,0.026,1)$ model exhibits the lowest value 0.57669 of AIC and is selected as the candidate model to forecast the volatility of stock returns of CHT Inc.

Finally, this study employs in-sample data and the estimated parameters to forecast the volatility of VACDGARCH type models and to compare the relative prediction performance of volatility. To assess the relative predication performance of volatility, this study employs the criteria of MAE and RSME. The empirical results of the forecasting indictor of VACD based GARCH-type models are presented in Table 8. As indicated in Table 8, the empirical results reveal that the VACD $(2,1)-\operatorname{IGARCH}(1,1)$ model, which has the relative lowest values $(0.0731$ and 0.1012$)$ of MAE and RMSE, respectively, exhibits relatively better performance of prediction on capturing volume duration.
Table 7: The Estimated Parameters of VACD-IGARCH Model of Volume Duration

\begin{tabular}{|c|c|c|c|c|}
\hline & $\begin{array}{c}\text { VACD } \\
(2,1)- \\
\text { IGARCH } \\
(1,1)\end{array}$ & $\begin{array}{c}\text { VACD }(2,1)- \\
\text { IGARCH } \\
(1,2)\end{array}$ & $\begin{array}{l}\text { VACD }(2,1)- \\
\text { IGARCH } \\
(2,1)\end{array}$ & $\begin{array}{c}\text { VACD } \\
(2,1)- \\
\text { IGARCH } \\
(2,2)\end{array}$ \\
\hline \multirow[t]{2}{*}{$w$} & $0.0044 * * *$ & $\begin{array}{c}0.0050 \\
* * *\end{array}$ & $\begin{array}{c}0.0003 \\
* * *\end{array}$ & $0.0034 * * *$ \\
\hline & $(0.0000)$ & $(0.0000)$ & $(0.0000)$ & $(0.0000)$ \\
\hline \multirow[t]{2}{*}{$\alpha_{1}$} & $\begin{array}{c}0.0054 \\
* * *\end{array}$ & $\begin{array}{c}0.0057 \\
* * *\end{array}$ & $0.0148 * * *$ & $\begin{array}{c}0.0217 \\
* * *\end{array}$ \\
\hline & $(0.0000)$ & $(0.0000)$ & $(0.0000)$ & $(0.0000)$ \\
\hline \multirow[t]{2}{*}{$\alpha_{2}$} & & $\begin{array}{c}0.0066 \\
* * *\end{array}$ & & $\begin{array}{c}0.0195 \\
* * *\end{array}$ \\
\hline & & $(0.0000)$ & & $(0.0000)$ \\
\hline \multirow[t]{2}{*}{$\beta_{1}$} & $\begin{array}{c}0.9946 \\
* * *\end{array}$ & $\begin{array}{c}0.9878 \\
* * *\end{array}$ & $\begin{array}{c}0.5448 \\
* * *\end{array}$ & $\begin{array}{c}0.4640 \\
* * *\end{array}$ \\
\hline & $(0.0000)$ & $(0.0000)$ & $(0.0000)$ & $(0.0000)$ \\
\hline \multirow[t]{2}{*}{$\beta_{2}$} & & & $\begin{array}{c}0.4404 \\
* * *\end{array}$ & $\begin{array}{c}0.4948 \\
* * *\end{array}$ \\
\hline & & & $(0.0000)$ & $(0.0000)$ \\
\hline \multirow[t]{2}{*}{$\gamma$} & $\begin{array}{c}0.0027 \\
* * *\end{array}$ & $\begin{array}{c}0.0029 \\
* * *\end{array}$ & $\begin{array}{c}0.0010 \\
* * *\end{array}$ & $\begin{array}{c}0.0005 \\
* * *\end{array}$ \\
\hline & $(0.0000)$ & $(0.0000)$ & $(0.0000)$ & $(0.0000)$ \\
\hline AIC & 0.3130 & 0.7152 & 0.3851 & 0.3349 \\
\hline
\end{tabular}

Note: $1 .{ }^{* * *}$ represents significant at the $1 \%$ level of significance.

Table 8: The Estimated Parameters of VACD-FIGARCH Model of Volume Duration $\varphi(L)(1-L)^{d} \varepsilon_{t}^{2}=\omega+[1-\beta(L)] u_{t}+\gamma x^{-1}$

\begin{tabular}{|c|c|c|c|c|c|c|c|}
\hline & $\begin{array}{c}\text { VACD }(2,1)- \\
\text { FIGARCH } \\
(1, d, 1)\end{array}$ & $\begin{array}{c}\text { VACD }(2,1)- \\
\text { FIGARCH } \\
(1, d, 2)\end{array}$ & $\begin{array}{c}\text { VACD }(2,1)- \\
\text { FIGARCH } \\
(2, d, 1)\end{array}$ & $\begin{array}{c}\text { VACD }(2,1)- \\
\text { FIGARCH } \\
(2, d, 2)\end{array}$ & $\begin{array}{c}\text { VACD }(2,1)- \\
\text { FIGARCH } \\
(3, d, 1)\end{array}$ & $\begin{array}{c}\text { VACD }(2,1)- \\
\text { FIGARCH } \\
(3, d, 2)\end{array}$ & $\begin{array}{c}\text { VACD }(2,1)- \\
\text { FIGARCH } \\
(3, d, 3)\end{array}$ \\
\hline \multirow[t]{2}{*}{$w$} & $\begin{array}{c}0.0337 \\
* * *\end{array}$ & $\begin{array}{c}0.0336 \\
* * *\end{array}$ & $\begin{array}{c}0.0158 \\
*\end{array}$ & 0.0085 & 0.0035 & 0.0000 & 0.0000 \\
\hline & $(0.0000)$ & $(0.0002)$ & $(0.0758)$ & $(0.3503)$ & $(0.7119)$ & $(1.0000)$ & $(1.0000)$ \\
\hline \multirow[t]{2}{*}{$\alpha_{1}$} & 0.0000 & 0.0000 & 0.0000 & 0.0000 & 0.0000 & 0.0000 & 0.0000 \\
\hline & $(1.0000)$ & (1.0000) & $(1.0000)$ & $(1.0000)$ & $(1.0000)$ & (1.0000) & (1.0000) \\
\hline \multirow[t]{2}{*}{$\alpha_{2}$} & & 0.0000 & & $0.0310 * *$ & & $0.0249 *$ & $0.0249 * *$ \\
\hline & & $(1.0000)$ & & $(0.0188)$ & & $(0.0652)$ & $(0.0650)$ \\
\hline \multirow[t]{2}{*}{$\alpha_{3}$} & & & & & & & 0.0000 \\
\hline & & & & & & & $(1.0000)$ \\
\hline$d$ & $\begin{array}{c}0.0078 \\
* *\end{array}$ & $\begin{array}{c}0.0078 \\
*\end{array}$ & $\begin{array}{c}0.0183 \\
* * *\end{array}$ & $\begin{array}{c}0.0175 \\
* * *\end{array}$ & $\begin{array}{c}0.0259 \\
* * *\end{array}$ & $\begin{array}{c}0.0240 \\
* * *\end{array}$ & $\begin{array}{c}0.0240 \\
* * *\end{array}$ \\
\hline
\end{tabular}




\begin{tabular}{|c|c|c|c|c|c|c|c|}
\hline & $(0.0322)$ & $(0.0731)$ & $(0.0002)$ & $(0.0004)$ & $(0.0000)$ & $(0.0001)$ & $(0.0001)$ \\
\hline \multirow[t]{2}{*}{$\beta_{1}$} & 0.0138 & 0.0138 & 0.0274 & $\begin{array}{c}0.0268 \\
*\end{array}$ & $\begin{array}{c}0.0368 \\
* *\end{array}$ & $\begin{array}{c}0.0344 \\
* *\end{array}$ & $\begin{array}{c}0.0344 \\
* *\end{array}$ \\
\hline & $(0.4442)$ & $(0.4481)$ & $(0.1174)$ & $(0.0991)$ & $(0.0318)$ & $(0.0351)$ & $(0.0361)$ \\
\hline \multirow[t]{2}{*}{$\beta_{2}$} & & & $\begin{array}{c}0.0175 \\
* * *\end{array}$ & $\begin{array}{c}0.0549 \\
* * *\end{array}$ & $\begin{array}{c}0.0217 \\
* * *\end{array}$ & $\begin{array}{c}0.0510 \\
* * *\end{array}$ & $\begin{array}{c}0.0510 \\
* * *\end{array}$ \\
\hline & & & $(0.0001)$ & (0.0009) & $(0.0000)$ & $(0.0023)$ & $(0.0022)$ \\
\hline \multirow[t]{2}{*}{$\beta_{3}$} & & & & & $\begin{array}{c}0.0114 \\
* * *\end{array}$ & $\begin{array}{c}0.0098 \\
* * *\end{array}$ & 0.0098 \\
\hline & & & & & $(0.0032)$ & $(0.0099)$ & $(0.5751)$ \\
\hline \multirow[t]{2}{*}{$\gamma$} & $\begin{array}{c}0.2749 \\
* * *\end{array}$ & $\begin{array}{c}0.2749 \\
* * *\end{array}$ & $\begin{array}{c}0.2746 \\
* * *\end{array}$ & $\begin{array}{c}0.2748 \\
* * *\end{array}$ & $\begin{array}{c}0.2745 \\
* * *\end{array}$ & $\begin{array}{c}0.2746 \\
* * *\end{array}$ & $\begin{array}{c}0.2746 \\
* * *\end{array}$ \\
\hline & $(0.0000)$ & $(0.0000)$ & $(0.0000)$ & $(0.0000)$ & $(0.0000)$ & $(0.0000)$ & $(0.0000)$ \\
\hline AIC & 0.57719 & 0.57735 & 0.57680 & 0.57674 & 0.57669 & 0.57671 & 0.57687 \\
\hline
\end{tabular}

Note: $1 .{ }^{*}, * *$ and ${ }^{* * *}$ represents significant at $10 \%, 5 \%$ and $1 \%$ level of significance, respectively.

Table 9: The Forecasting Indictor of VACD Based GARCHType Models

\begin{tabular}{|c|c|c|}
\hline & MAE & RMSE \\
\hline VACD $(2,1)-\operatorname{GARCH}(2,1)$ & 0.0840 & 0.1471 \\
\hline VACD $(2,1)-\operatorname{IGARCH}(1,1)$ & 0.0731 & 0.1012 \\
\hline VACD $(2,1)-$ FIGARCH $(3,0.026,1)$ & 0.4234 & 0.6119 \\
\hline
\end{tabular}

\section{Conclusions}

In general financial theory, when investors obtain private news and carry on their investment strategy, it would exhibit large amount of transaction. The block trading volume would have great impact on the change of stock price. Thus, the purpose of this study is to construct ACD model for the block trading duration. The ACD model based on the block trading volume duration is referred to as Volume ACD (VACD) in this study. This study selects Chunghwa Telecom (CHT) Inc., offering the America Depository Receipt (ADR) in NYSE, to investigate the block trading volume duration in Taiwanese equity market. The VACD model is further integrated into GARCH-type models to capture the property of the long-term memory in volatility. Therefore, the VACD-GARCH type models employed in this study include VACD-GARCH, VACDIGARCH and VACD-FIGARCH models.

The empirical results of this study indicate that VACD $(2,1)-G A R C H \quad(2,1)$, VACD $(2,1)-I G A R C H \quad(1,1)$ and VACD (2,1)-FIGARCH $(3,0.026,1)$ are relatively more appropriate to capture the volume duration clustering of CHT Inc., respectively. The reciprocal of duration is significantly positive in all specifications, indicating that the longer the volume duration, the smaller the volatility of stock price and vice versa. This empirical result also reveal that the frequency of transactions carries information about the transaction clustering proposed by Easley and O'Hara (1992). The long memory in volume duration series increases dependence at level of volatility clustering. The volume duration of interdependence can be an effect of long memory in volatility, and the long memory in volatility is corresponding for changing in dependence structure. Moreover, the VACD (2,1)-IGARCH $(1,1)$ exhibits relatively better performance of prediction on capturing volume duration. This volatility model is more appropriate in this study to portray the changes of the CHT Inc. prices and provides more information about the volatility process for investing strategy, which can be a reference indicator of financial asset pricing, hedging strategy and risk management.

\section{References}

Aitken, M., \& Frino, A. (2005). Asymmetry in Stock Returns Following Block Trades on the Australian Stock Exchange: A Note. Journal of Accounting, Finance and Business, 32(1), 5461.

Akaike, H. (1976). Canonical Correlation Analysis of Time Series and the Use of an Information Criterion. In R. Metha \& K. Lainiotis (eds), System Identification: Advances and Case Studies. New York, NY: Academic Press, Inc.

Baillie, R. T., Bollerslev, T., \& Mikkelsen, H. O. (1996). Fractionally Integrated Generalized Autoregressive Conditional Heteroskedasticity. Journal of Econometrics, 74, 3-30.

Bollerslev, T. (1986). Generalized Autoregressive Conditional Heteroskedasticity. Journal of Econometrics, 31, 307-327.

Easley, D., \& O’Hara, M. (1987). Price, Trade Size and Information in Securities Markets. Journal of Financial Economics, 19, 69-90.

Easley, D., \& O'Hara, M. (1992). Time and the process of security 
price adjustment. Journal of Finance, 47, 905-927.

Engle, R. F. (1982). Autoregressive Conditional Heteroskedasticity with Estimates of the Variance of U.K. Inflation. Econometrica, 50, 987-1008.

Engle, R. F., \& Bollerslev, T. (1986). Modelling the Persistence of Conditional Variances. Econometric Reviews, 5, 81-87.

Engle, R. F., \& Russell, J. R. (1998). Autoregressive Conditional Duration: A New Model for Irregularly Spaced Transaction Data. Econometrica, 66, 1127-1162.

Engle, R. F. (2000). The Econometrics of Ultra-High-Frequency Data. Econometrica, 68, 1-22.

Gallant, A. R., Rossi, P. E., \& Tauchen, G. (1992). Stock Prices and Volume. Review of Financial Studies, 2, 133-149.

Go, Y.-H., \& Lau, W.-Y. (2016). Information Arrival between Price Change and Trading Volume in Crude Palm Oil Futures Market: A Non-linear Approach. Journal of Asian Finance, Economics and Business, 3(3), 79-91. https://doi.org/10.13106/jafeb.2016.vol3.no3.79.

Heynen, R. C., \& Kat, H. M. (1994). Volatility Prediction: A Comparison of the Stochastic Volatility, GARCH $(1,1)$ and EGARCH $(1,1)$ Models. Journal of Derivatives, 2, 50-65.

Jati, K., \& Premaratne, G. (2017). Analysis of Staple Food Price Behaviour: Multivariate BEKK-GARCH Model. Journal of Asian Finance, Economics and Business, 4(4), 27-37. http://dx.doi.org/10.13106/jafeb.2017.vol4.no4.27

Kraus, A., \& Stoll, H. R. (1972). Price Impact of Block Trading on the New York Stock Exchange. Journal of Finance, 27, 569-588.
Lumsdaine, R. L. (1992). Finite Sample Properties of the Maximum Likelihood Estimator in GARCH $(1,1)$ and IGARCH (1,1) Models: A Monte Carlo Investigation (Unpublished Manuscript). Department of Economics, Princeton University.

Meitz, M., \& Terasvirta, T. (2006). Evaluating Models of Autoregressive Conditional Duration. Journal of Business \& Economic Statistics, 24(1), 104-124.

Mendes, B. V. M., \& Kolev, N. (2008). How Long Memory in Volatility Affects True Dependence Structure. International Review of Financial Analysis, 17, 1070-1086.

Ng, W. L. (2008). Analysing Liquidity and Absorption Limits of Electronic Markets with Volume Duration. Quantitative Finance, 8(4), 353-361.

Nguyen, C. T., \& Nguyen, M. H. (2019). Modeling Stock Price Volatility: Empirical Evidence from the Ho Chi Minh City Stock Exchange in Vietnam. Journal of Asian Finance, Economics and Business, 6(3), 19-26. https://doi.org/10.13106/jafeb.2019.vol6.no3.19

Racicot, F. E., Théoret, R., \& Coen, A. (2008). Forecasting Irregularly Spaced UHF Financial Data: Realized Volatility vs UHF-GARCH Models. International Advance in Economic Research, 14, 112-124.

Sahadudheen, I. (2015). An Exponential GARCH Approach to the Effect of Impulsiveness of Euro on Indian Stock Market. Jour nal of Asian Finance, Economics and Business, 2(3), 17-22. https://doi.org/10.13106/jafeb.2015.vol2.no3.17.

Shome, D. K., \& Singh, S. (1995). Firm Value and External Blockholdings. Financial Management, 24(4), 3-14. 